\title{
Magnetic resonance arthrography of the hip: technique and spectrum of findings in younger patients
}

\author{
Sébastien Aubry • Danny Bélanger • Caroline Giguère • \\ Martin Lavigne
}

Received: 18 February 2010/Revised: 26 April 2010 /Accepted: 28 April 2010 /Published online: 8 June 2010

(C) European Society of Radiology 2010

\begin{abstract}
Magnetic resonance(MR) imaging is the reference imaging technique in the evaluation of hip abnormalities. However, in some pathological conditions - such as lesions of the labrum, cartilaginous lesions, femoroacetabular impingement, intra-articular foreign bodies, or in the pre-operative work-up of developmental dysplasia of the hip-intraarticular injection of a contrast medium is required to obtain a precise diagnosis. This article reviews the technical aspects, contraindications, normal appearance and potential pitfalls of MR arthrography, and illustrates the radiological appearance of commonly encountered conditions.
\end{abstract}

Keywords Magnetic resonance arthography $\cdot$ MRI $\cdot$ Hip

\section{Introduction}

The development of magnetic resonance (MR) imaging has allowed it to become the standard of reference in the work-up of bone and soft tissue abnormalities of the hip. However, some pathological conditions - such as lesions

S. Aubry $(\bowtie)$

Service de Radiologie A., CHU Jean Minjoz,

Boulevard Flemming,

25000 Besançon Cedex, France

e-mail: radio.aubry@free.fr

S. Aubry $\cdot$ D. Bélanger $\cdot$ C. Giguère

Département de Radiologie, Université de Sherbrooke,

Sherbrooke, QC, Canada

M. Lavigne

Département d'Orthopédie, Hôpital Maisonneuve Rosemont,

Montréal, QC, Canada of the labrum, cartilaginous lesions, femoroacetabular impingement, intra-articular foreign bodies or in the preoperative work-up of developmental dysplasia of the hiprequire intra-articular injection of a contrast medium, i.e. to perform MR arthrography, in order to obtain a precise diagnosis. This article's objectives are to refresh the technical aspects, contraindications, normal appearance and potential pitfalls of MR arthrography; and in addition, to illustrate the radiological findings of commonly encountered conditions.

\section{Technique}

Intra-articular access

In our institution, MR arthrography involves two steps: first, intra-articular injection under fluoroscopic guidance, then MR imaging. The different steps for intra-articular injection are as follows:

- Putting the patient in a dorsal decubitus position, the lower limb at 10-15 degrees of internal rotation, with the tube being positioned vertically

- Homing in on the injection site under fluoroscopic guidance

- Aseptic preparation of the skin

- Placement of a sterile field

- Local subcutaneous anaesthesia with lidocaine $1 \%$. Up to $4 \mathrm{ml}$ may be injected, but subcutaneous anaesthesia is not absolutely necessary and would be discussed with the patient

- Intra-articular access under fluoroscopic guidance with a 22-gauge spinal needle 
- intra-articular injection under fluoroscopic guidance, $12 \mathrm{ml}$ of iodinated contrast medium (Iodixanol Visipaque $270 \mathrm{mg} \mathrm{I} / \mathrm{ml}$, GE Healthcare) mixed with $0.06 \mathrm{ml}$ gadolinium chelates (Gadopentetate Dimeglumine Magnevist $0.5 \mathrm{mmol} / \mathrm{ml}$, Bayer) [1] or $10-15 \mathrm{ml}$ from a prefilled syringe of gadolinium chelates (Gadoteric acid Artirem $0.0025 \mathrm{mmol} / \mathrm{ml}$ Guerbet)

The cutaneous access location is found lateral to the femoral vessels and under the inguinal ligament. It is chosen at the preference of the operator, generally at the superior or middle part of the femoral anatomical neck (Fig. 1). Injection at the superior part of the neck may cause less pain, but would risk more extra-articular leakage of contrast medium compared with injection of the middle part [2]. MR imaging should ideally be performed less than 45 min after arthrography because of the decline of the signal-to-noise ratio (SNR) over time, although a 1-h delay may be tolerated $[3,4]$.

Miller proposed a technique consisting of a fluoroscopically guided landmarking of the cutaneous access point, then intra-articular access in the MR suite [5]. Intraarticular access may also be guided by MR fluoroscopy [6].

\section{MR apparatus}

MR operating systems nowadays have magnetic field strengths from 0.2-3 Tesla (T). Because it has a higher $\mathrm{SNR}$, a greater magnetic field (3 T) allows for higher spatial resolution in the same time period or faster acquisition with identical spatial resolution $[7,8]$. Evaluation of the labrum and cartilage would be better with a 3-T magnetic field [9]. Phased-array coils are needed for parallel imaging techniques [simultaneous acquisition of spatial harmonics (SMASH) or sensitivity encoding (SENSE)]. These reduce

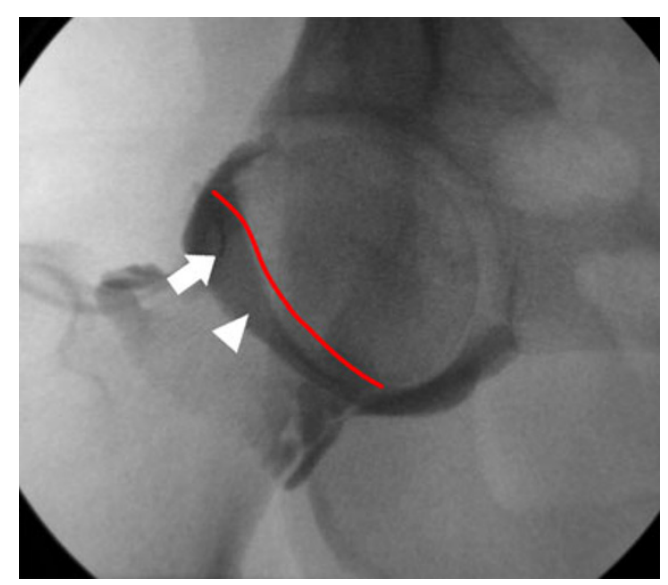

Fig. 1 Intra-articular injection in a right hip joint under fluoroscopic guidance. Puncture site (white arrow) is at the superior part of the anatomical neck of the femur but the middle part could have been chosen (white arrowhead) imaging time and the specific absorption rate [7, 10] with, according Ryan et al. [11], no discernible difference in image quality or SNR between images acquired with and without SENSE.

\section{MR sequences}

Our routine protocol (Table 1) for MR arthrography of the hip at $1.5 \mathrm{~T}$ includes fat-saturated T1-weighted 3-mm slices in axial oblique, coronal and sagittal planes; fatsaturated T2-weighted 4-mm slices in the coronal plane; and fat-saturated three-dimensional (3D) gradient echo T1weighted 1-mm slices allowing for multiplanar and radial reconstructions, which could help in the detection of acetabular labrum lesions [12] and improve the analysis in its weight-bearing region [13]. The field of view used is at a narrow 14-18 cm, focusing on the symptomatic hip. Some authors opt for fat-saturated proton density (PD)-weighted oblique axial slices parallel to the femoral neck to analyse the head-neck junction [14].

\section{Contraindications and precautions}

\section{Contraindications}

As with all MR studies, absolute contraindications related to magnetic field exposure need to be respected. Principally, these are implantable material (cardiac stimulators, neurostimulators, cochlear implants etc.), certain types of cardiac valves, ferromagnetic material and metallic foreign bodies. Osteosynthesis material is not an absolute contraindication to MR studies, but when situated in close proximity to the region of interest, magnetic susceptibility artefacts may worsen image quality to the extent of rendering image interpretation impossible. For that reason, we elect not to perform MR arthrography of the hip for patients with osteosynthesis material at the level of the cotyle or at the upper extremity of the homolateral femur.

Precautions related to intra-articular injection of contrast media

Intra-articular access is an invasive procedure, and systematic precautions have to be followed:

- Informing the patient about the different steps of the procedure, of potential allergic haemorrhagic or infectious complications, and obtaining consent

- Verifying absence of anticoagulation or anti-platelet aggregation medication

- Verifying the absence of allergies to the products used

When strict aseptic precautions are respected, infectious complications are avoided. Arthrography may be responsi- 
Table 1 Detailed parameters of our standard examination protocol

\begin{tabular}{|c|c|c|c|c|c|}
\hline Sequence & $\begin{array}{l}\text { Fat-saturated } \\
\text { T1-weighted }\end{array}$ & $\begin{array}{l}\text { Fat-saturated } \\
\text { T1-weighted }\end{array}$ & $\begin{array}{l}\text { Fat-saturated } \\
\text { T1-weighted }\end{array}$ & $\begin{array}{l}\text { Fat-saturated } \\
\text { T2-weighted }\end{array}$ & $\begin{array}{l}\text { Fat-saturated 3D gradient } \\
\text { echo T1-weighted }\end{array}$ \\
\hline Orientation & Axial oblique & Sagittal & Coronal & Coronal & Axial \\
\hline Slice thickness $(\mathrm{mm})$ & 3 & 3 & 3 & 4 & 1 \\
\hline Slice gap (mm) & 1 & 1 & 1 & 1 & 0.2 \\
\hline Number of slices & 20 & 21 & 19 & 19 & 80 \\
\hline $\mathrm{TR}(\mathrm{ms})$ & 644 & 683 & 634 & 4000 & 12,6 \\
\hline $\mathrm{TE}(\mathrm{ms})$ & 12 & 12 & 12 & 68 & 6.21 \\
\hline Flip angle (degree) & 180 & 180 & 180 & 180 & 14 \\
\hline Acquisition matrix size (pixels) & $384 \times 256$ & $384 \times 256$ & $384 \times 256$ & $512 \times 256$ & $256 \times 217$ \\
\hline Field of view (mm) & 180 & 180 & 180 & 385 & 180 \\
\hline
\end{tabular}

ble for a temporary flare-up of joint pain, reaching its peak $4 \mathrm{~h}$ post-injection, independent of the quantity of local anaesthetic previously injected [15]. Minor adverse reactions are rarely reported, such as headache, vertigo, vasovagal episode or urticaria [16], but Saupe and co-workers' series of 1,085 MR arthrographies revealed no major adverse reactions [15].

\section{Normal anatomy and variants}

\section{Normal anatomy of the hip}

The hip joint is an enarthrosis joining the femoral head with the iliac bone (Fig. 2). The articular surfaces are formed by the femoral head, a sphere of $45 \mathrm{~mm}$ in diameter; and the acetabulum, a semi-circular cavity in the shape of a

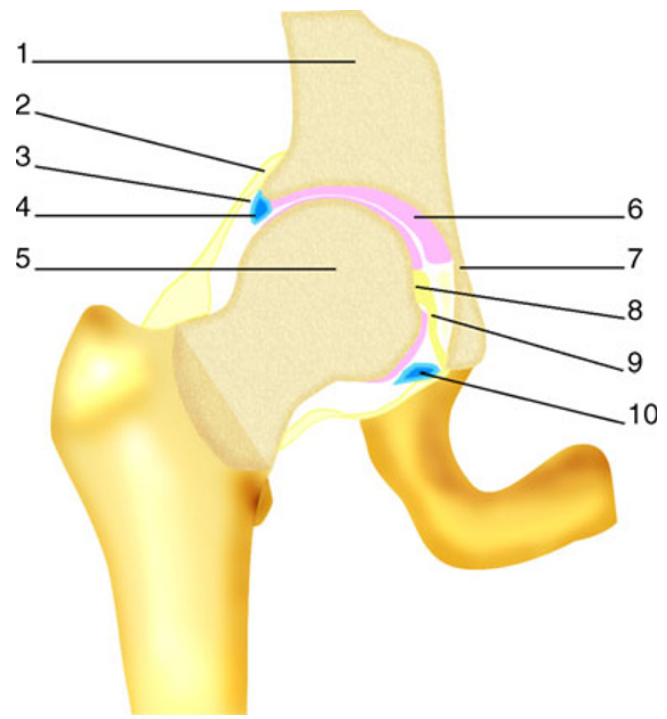

Fig. 2 Hip joint anatomy. 1 Ilium, 2 capsula articularis, 3 perilabral recess, 4 acetabular labrum, 5 head of femur, 6 articular cartilage, 7 acetabulum, 8 fovea, 9 ligamentum teres, 10 transverse ligament horse-shoe closed off inferiorly by the transverse ligament. These are covered by an articular cartilage 1-3 mm thick $[17,18]$ except at the centre of the acetabulum and at the level of the fovea, where the ligament of the femoral head (ligamentum teres) is inserted. The acetabular labrum is a triangular fibrocartilage inserted at the periphery of the acetabulum (Fig. 2). It increases the depth of the acetabulum [19], and is thicker in its postero-superior compared with its antero-inferior aspect [20, 21]. The articular capsule forms a fibrous sleeve around the whole joint between the base of the labrum and the femoral neck. Normally, there is a small perilabral recess between the labrum and the capsule, shown with contrast medium [22] (Fig. 3). On axial slices, the articular capsule rests in front of the iliofemoral ligament and the ischiofemoral ligament, at the back. The normal hip joint capacity is $8-20 \mathrm{ml}[23,24]$. Located anteriorly, the ilio-psoas bursa communicates with the hip joint in 10-15\% of cases [25] (Fig. 4).

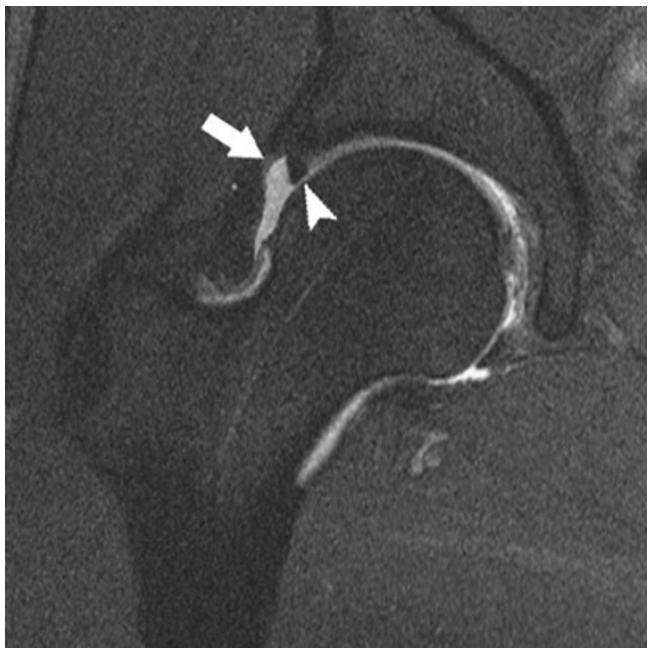

Fig. 3 Normal hip joint. Coronal fat-saturated T1-weighted arthroMR image demonstrates a perilabral recess (white arrow). The superior labrum has a normal triangular shape and an homogeneous low signal (white arrowhead) 


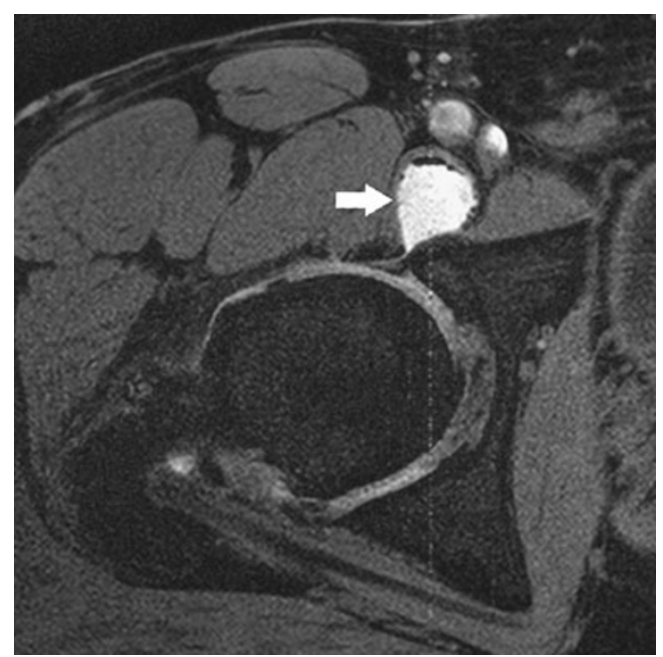

Fig. 4 Opacified ilio-psoas recess and osteoarthritis in a 45-year-old man. Axial fat-saturated T1-weighted 3D gradient echo image shows the ilio-psoas recess filled by contrast medium and containing some small post-injection bubbles (white arrow). Moreover, heterogeneous signal of the labrum and pericephalic osteophytes are consistent with osteoarthritis

Normal variants and pitfalls

Anatomical variants of the labrum have been described and they should not be interpreted falsely as abnormal:

- The junctional zone between the acetabular labrum and the transverse ligament is generally indistinguishable. An irregularity of this zone is possible and may give a pseudotear aspect to the labrum [26].

- Most often the labrum is triangular in shape, but it may be rounded or flat in asymptomatic patients [27, 28]. It appears that with age, even in the absence of symptoms, the proportion of triangular labra diminishes $[29,30]$, and intralabral T1 intermediate and T2 hypersignal anomalies appear [27, 30]. Complete absence of the labrum is possible $[26,28,30]$ : the antero-superior labrum may not be visualised in $10 \%$ of cases; this proportion increases with age [28].

- The existence of a sublabral recess is possible (Fig. 5). In MR imaging, it appears as a well-defined linear interposition of contrast medium partially separating the underside of the labrum without any perilabral anomalies [31]. However, many locations have been described: Petersilge et al.n [24] believe it is always in an anterosuperior position; on the other hand, Studler et al. [31] describe it in an antero-inferior location. Dinauer et al. [32] also reported the possibility of a postero-inferior sublabral recess.

- There is a possibility of a sulcus between the labrum and the articular cartilage at the level of the acetabular fossa $[20,33]$.

\section{Abnormality of the acetabular labrum}

Abnormality of the acetabular labrum may be traumatic, degenerative or related to hip dysplasia [34-36]. The clinical presentation typically consists of clicking, locking and pain on flexion and internal rotation of the hip. Conventional MR imaging of the hip is not effective, except in the study by Mintz et al. [37], in the evaluation of labral tears, while MR arthrography has shown good sensitivity and specificity in the detection of acetabular labral tears [22, 24, 26, 33, 38, 39]. Acetabular labral lesions are considered to be precursors of coxarthrosis. Early diagnosis and precise determination of the location and extent of labral tears may allow for a timely surgical intervention [22].

In 1996, Czerny et al. [33] proposed the following MR imaging classification of acetabular labrum lesions in increasing stages of severity:

- Stage 0 corresponds to a normal labrum

- Stage 1A corresponds to the presence of an intra-labral increased T2 signal not in continuity with the labral margin of a triangular labrum

- Stage 2A corresponds to a partial labral tear with extension of contrast media into an undetached triangular labrum

- Stage 3A corresponds to a complete labral tear through a triangular labrum

- In Stages 1B, 2B and 3B, the labrum is thickened and a labral recess is not present

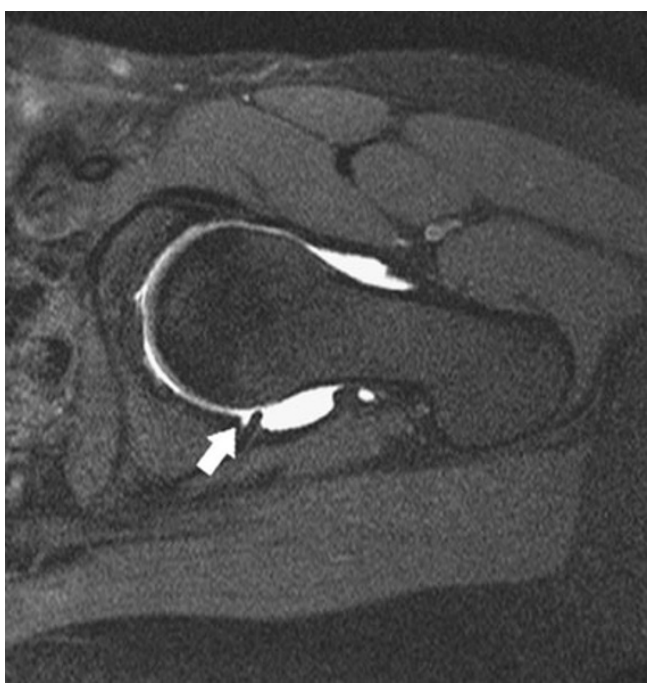

Fig. 5 Normal variant of the posterior labrum. On this axial oblique fat-saturated T1-weighted image of a 17-year-old girl's hip, a linear interposition of contrast medium separates partially the underside of the posterior labrum (white arrow), consistent with a posterior sublabral recess. The signal of the labrum is normal, and its rounded shape is also a normal variant 
However, Blankenbaker et al. [40] did not find a good correlation between Czerny et al.'s classification and Lage et al.'s arthroscopic classification [41]. The author proposed to describe the morphology of labral tears rather than using a specific classification scheme:

- An irregular labrum, i.e. irregular margins without a tear (Fig. 6a)

- A flap-type labral tear, may be partial or complete (Fig. 6b)

- A longitudinal peripheral labral tear, located at the base of the labrum, may be partial (Fig. 6c) or complete (Fig. 6d)

- A thickened and distorted labrum, in keeping with the instability of the labral lesion

A linear or curvilinear increased signal at the insertion of the labrum is compatible with a partial tear, but needs to be interpreted with caution, in light of other clinical information, as it can be found in asymptomatic patients (Fig. 7): in a series of 52 examinations in asymptomatic patients, Cotten

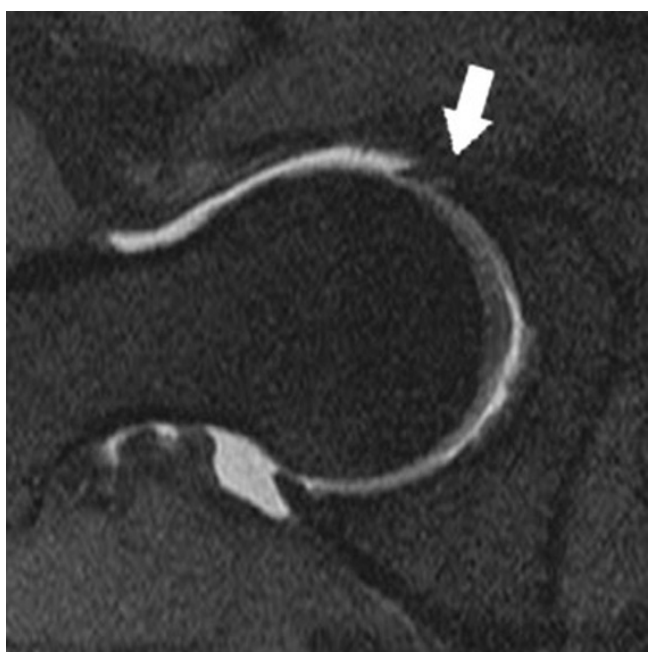

Fig. 7 Axial fat-saturated T1-weighted arthro-MR image. This curvilinear hyperintense line visible on the articular side of the anterior labrum (white arrow) consistent with a partial tear can be asymptomatic
Fig. 6 Elementary labral lesions on fat-saturated T1-weighted arthro-MR images. a Axial oblique image. Irregular anterior labrum appearing of undefined degenerative high signal, without formal evidence of tear. b Coronal image. An horizontal well-defined high-signal line visible within the superior labrum is consistent with a flap tear of the labrum. Moreover, cartilaginous thinning, osteophytes and a tear of the superior capsule are detected. c Sagittal image shows a peripheral vertical linear hyperintense line in the labrum at 2 o'clock. As it communicates with the articular surface but not with the perilabral recess; it is consistent with a partial longitudinal labral tear. d Sagittal image shows a complete longitudinal labral tear at 2 o'clock
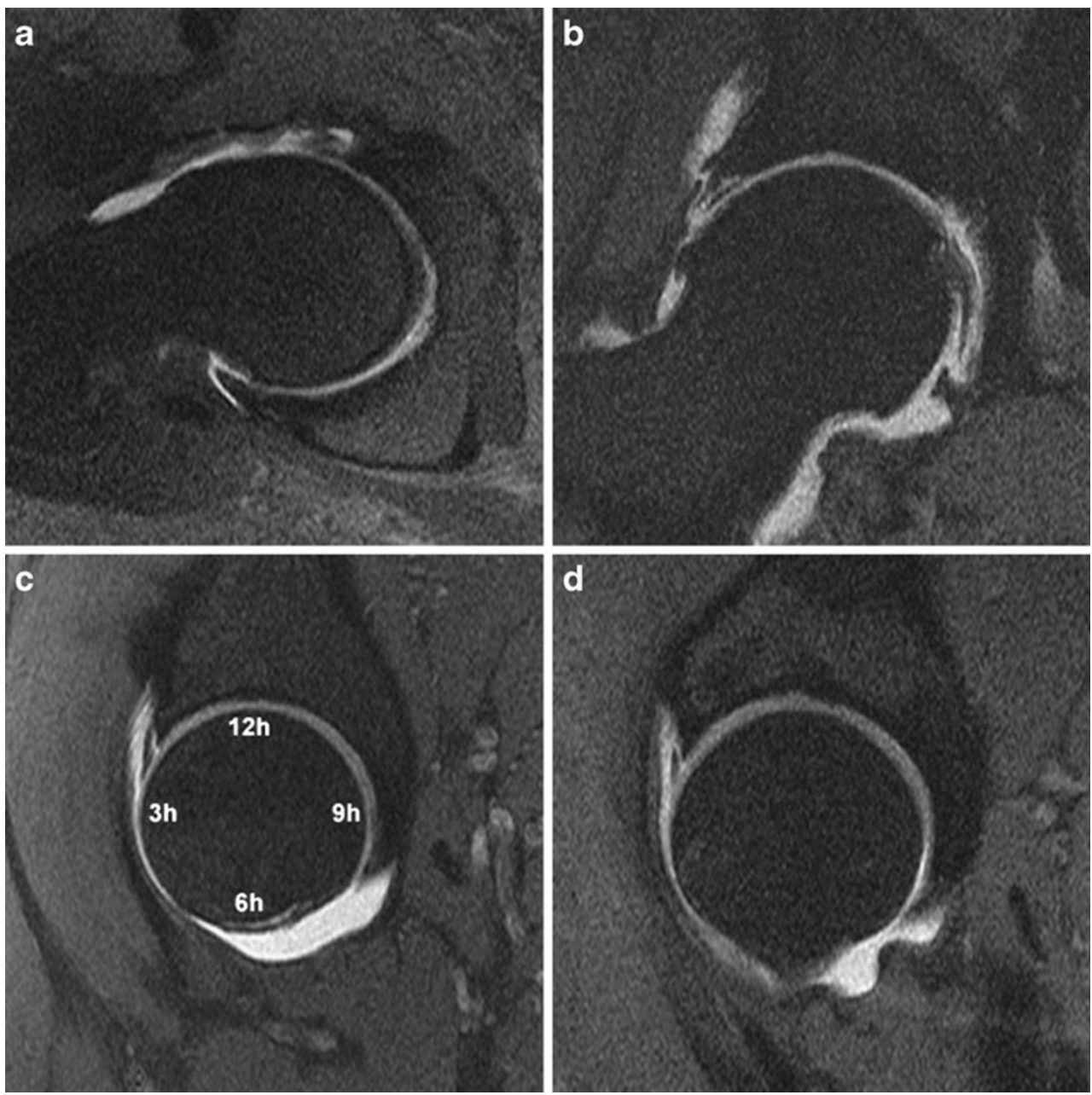
et al. [28] described four cases of labral tear (7.7\%). The presence of a paralabral cyst is an indirect sign of complete labral tear (Fig. 8) [42]. Paralabral cysts may lead to acetabular erosions [43] and appear to be more frequent in cases of developmental dysplasia of the hip [44].

In young patients presenting with pain, a diagnosis of developmental dysplasia of the hip on plain film is often associated on MR imaging with acetabular labrum abnormality showing hypertrophy, irregularity, intermediate or hypersignal (Fig. 9), an inverted horizontal or trapped appearance, and sometimes a tear. With time, direct contact between the abnormal labrum and the femoral chondral surface leads to the formation of bony protrusions and the equivalent of femoroacetabular impingement. Progressively, it evolves to coxarthrosis.

In $30 \%$ of cases, labral lesions are associated with chondropathy [45]. Most labral tears involve the anterior and superior labrum [41]. Using the clock-face method to localise and describe the extent of a labral tear is more precise, besides more than $40 \%$ of labral tears extend beyond a quadrant [41]: on a sagittal slice, 12 o'clock is above, 3 o'clock in front, 6 o'clock below and 9 o'clock behind (Fig. 6c).

\section{Femoroacetabular impingement}

Femoroacetabular impingement (FAI) is a cause of acetabular labrum abnormality and early coxarthrosis; and is potentially treatable surgically [46-49]. Clinically, as in labral tears, patients with FAI may complain of pain on hip flexion and internal rotation. A limited range of motion on internal rotation of the hip should also point to the

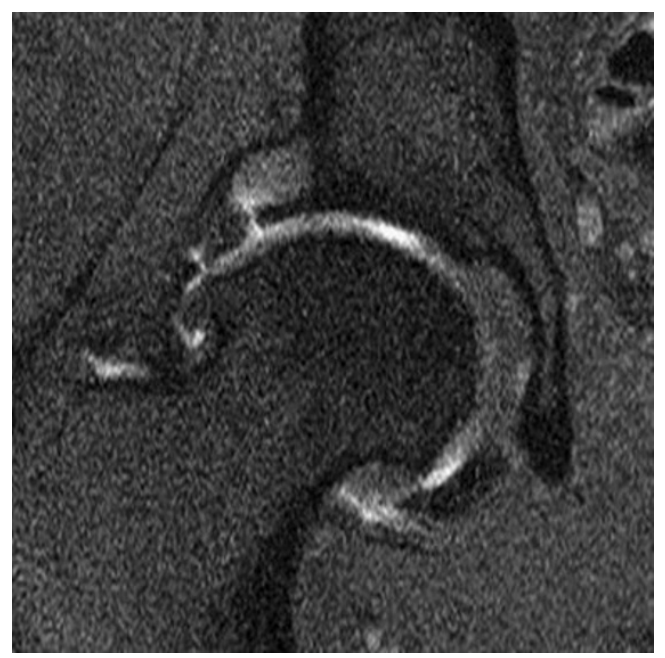

Fig. 8 Coronal reconstruction from a fat-saturated 3D gradient echo acquisition. A paralabral cyst is opacified by contrast media through a complete labral tear of the superior labrum

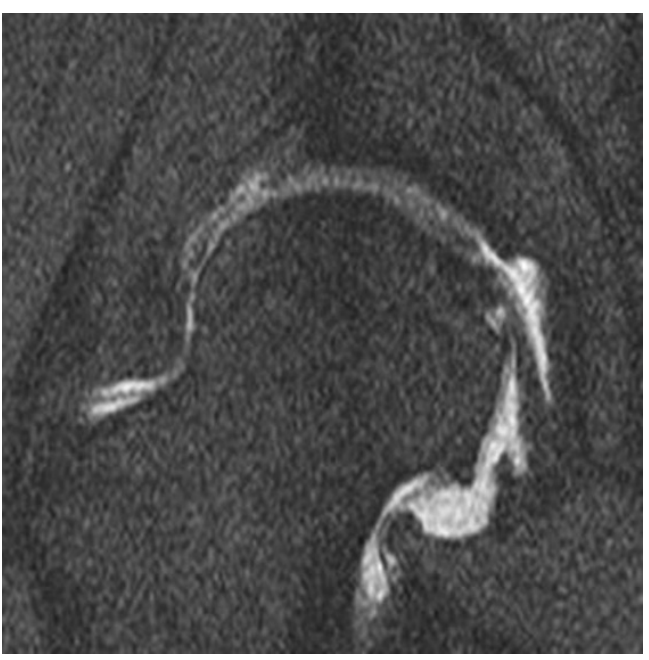

Fig. 9 This coronal fat-saturated T1-weighted arthro-MR image shows degenerative changes associated with a slight hip dysplasia: the superior labrum is irregular and of intermediate signal. Small pericephalic osteophytes are also present

diagnosis. Early on, radiographs may be normal or may show subtle anomalies: a prominent anterior portion of the head-neck junction, anterior synovial herniation pits, the presence of an os acetabuli, ossification of the acetabular border, coxa profunda, acetabular protrusion or acetabular retroversion $[48,50]$.

According to the mechanism thought to be responsible for each, FAI is classified into two types: the cam type, where the antero-superior part of the anatomical neck of the femur is hypertrophied and convex in shape impinging between the labrum and acetabulum; and the pincer type, where there is excessive coverage of the femoral head by the acetabulum (Fig. 10). The cam type is more frequent compared with the pincer type, but many cases of FAI are caused by both morphological anomalies and are not pure forms of either type [51].

\section{Cam-type FAI}

In this type of FAI, the so-called "pistol grip deformity" of the anterior portion of the femoral head-neck junction causes a compression and abnormal stress on the anterior labrocartilaginous complex on flexion and internal rotation. It is most frequently found in young athletic males $[46,47]$.

Cam-type FAI may be congenital or due to developmental dysplasia of the hip, slipped capital femoral epiphysis, trauma or avascular necrosis [14] but contrary to slipped capital femoral epiphysis and growth abnormalities of the femur congenital dysplasia is a rare cause of cam impingement because the head-neck junction in these patients is usually normal. 


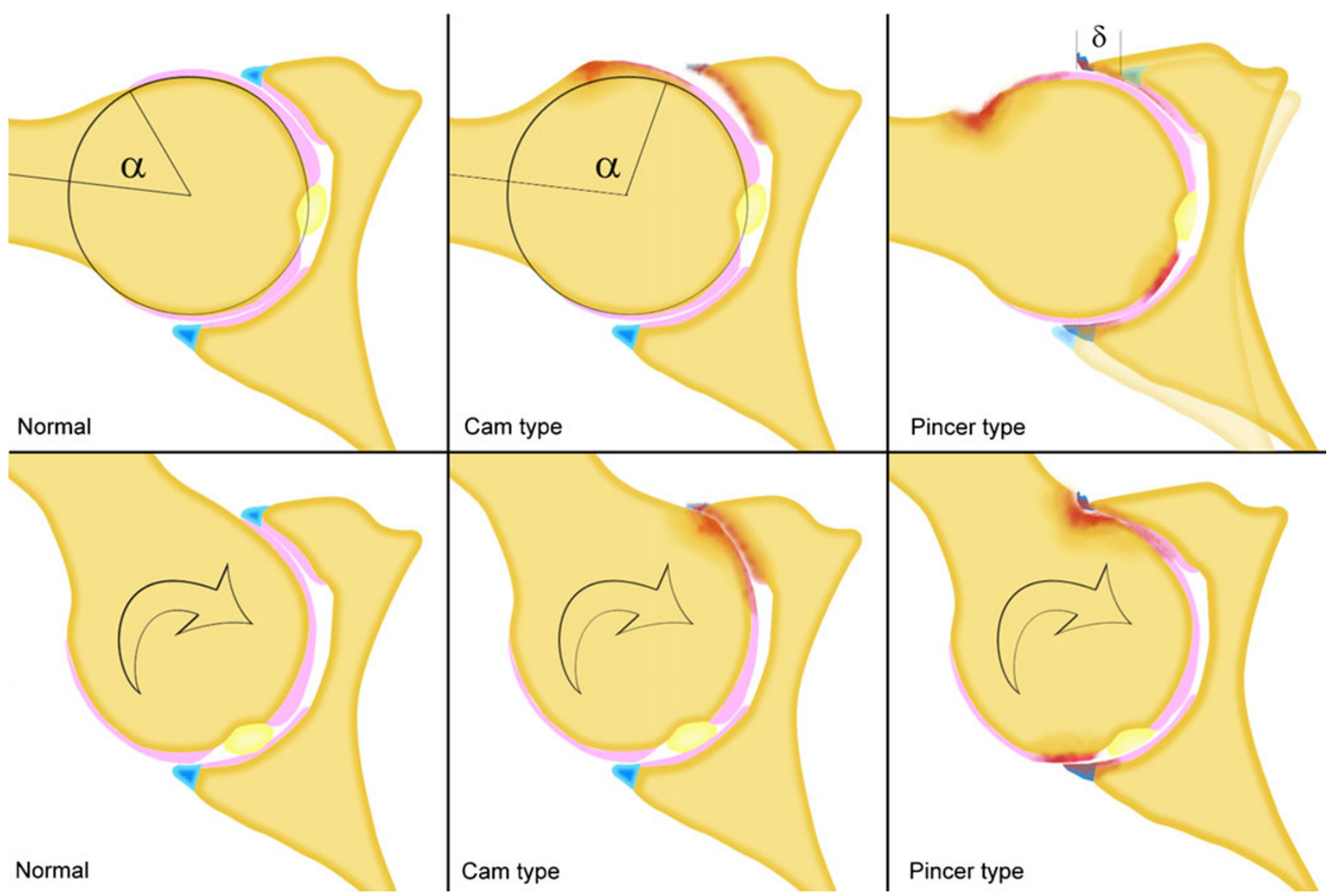

Fig. 10 Drawings of the pathophysiology of FAI. Left Normal hip: normal range of movement without impingement while medial rotation. Middle Cam-type of FAI: when hip rotates medially, the hypertrophied anterior head-neck junction bumps into the acetabulum

MR arthrography signs of cam-type FAI are:

- A flattening or a convexity of the antero-superior part of the normally concave femoral head-neck junction (Fig. 11) [49]. The so-called "pistol grip deformity" is the nickname for a pronounced deformity of the femur and should not be used for slighter deformities with abnormal head-neck offset.

- An alpha angle exceeding 55 degrees (Fig. 11) [52]. The alpha angle is measured on an axial-oblique image between the axis of the femoral neck and a line connecting the femoral head centre with the point of change in the radius of the curvature on the anterior part of the femoral head. Its normal value is 42 degrees. The pistol grip deformity increases the alpha angle. The alpha angle may be underestimated if the contour anomaly is anterosuperior or superior, some recommend measuring the alpha angle on radial reconstructions around the femoral neck axis [53].

- In general, an earlier, more severe and extended involvement of the antero-superior cartilage [54] com- and anterior labrum. Right Pincer-type of FAI: acetabular overcoverage causes early contact between the acetabulum and femoral neck, and contrecoup postero-inferior degenerative lesions

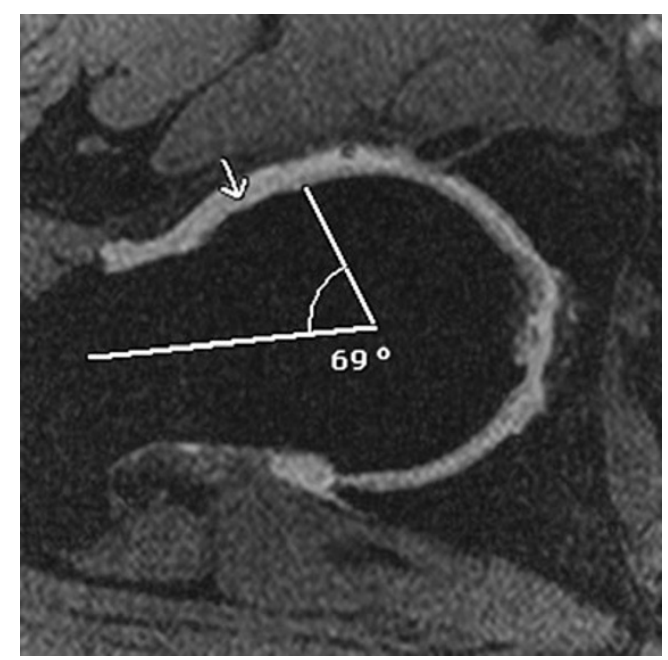

Fig. 11 Cam-type of FAI: anterior bulge of the head-neck junction (white arrow) and increased alpha angle (69 degrees vs 55 degrees maximum). Anterior labrum is torn off and calcified 

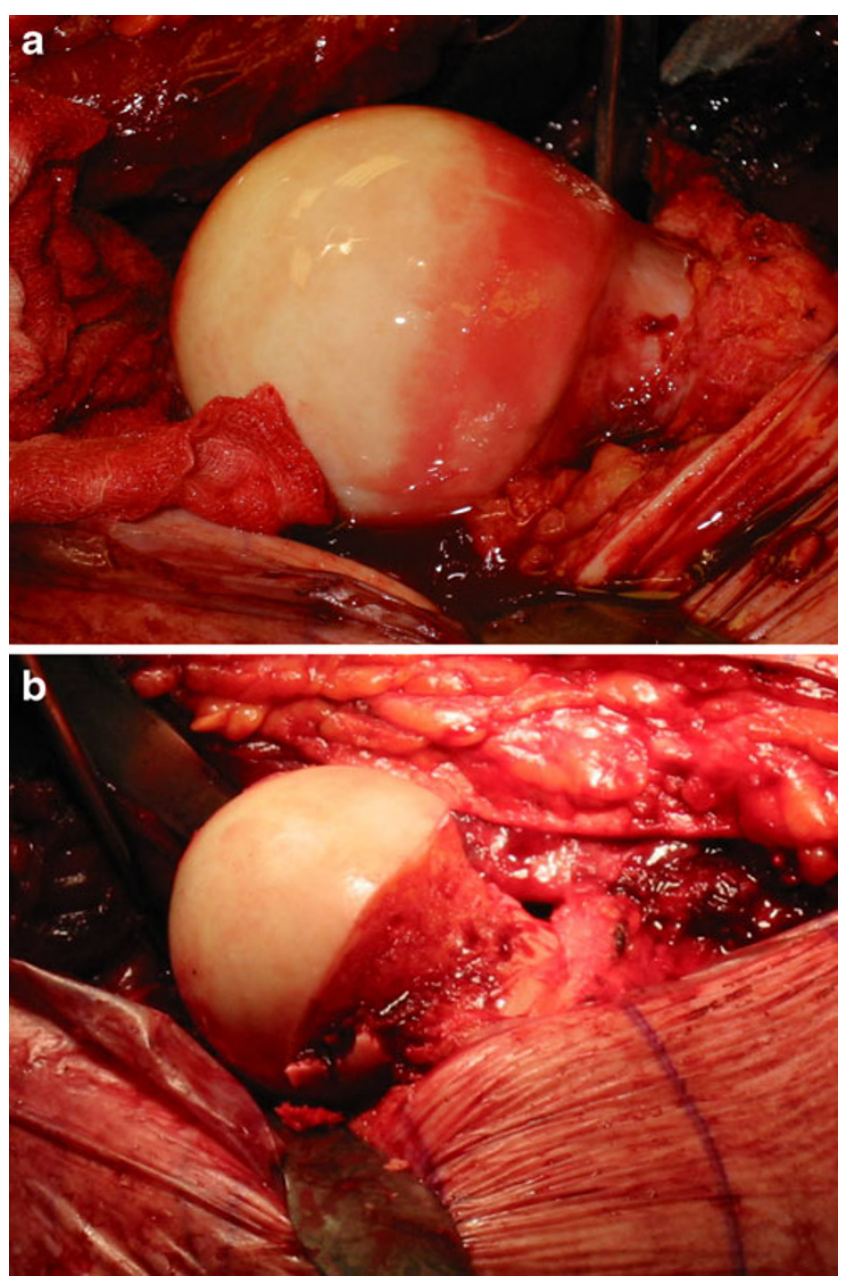

Fig. 12 a, b Intraoperative view during a surgical osteoplasty in the treatment of a cam-type of FAI. a Before osteoplasty, fibrocystic changes at the anterior aspect of the anatomical neck of the femur are visible. b After osteoplasty, the hypertrophic head-neck junction has been removed

pared with the pincer-type FAI [48]; and may be associated with subchondral oedema shown by high signal intensity on fat-saturated $\mathrm{T} 2$-weighted and fatsaturated PD-weighted sequences.

- There is often extensive antero-superior labral involvement $[48,54]$.

- Fibrocystic changes and subchondral microcysts in the antero-superior part of the femoral neck, not to be mistaken for physiological anterior synovial invaginations [55].

The therapeutic approach aims at early restoration of the normal morphology of the hip joint before the setting in of chondropathy and coxarthrosis. Some even recommend treating patients presenting with impaired flexion-internal rotation of the hip in association with MR imaging signs of FAI, without pain symptoms [56]. Surgical interventions for cam-type FAI include osteoplasty of the anterior portion of the femoral neck-head junction (Fig. 12), relieving the cause of impingement, arthroscopic debridement of labral tears and possible removal of foreign bodies [57]. On postoperative follow-up, an alpha angle of 43 degrees measured on standard plain films allows for FAI-free motion of the hip, but only MR arthroscopy with radial reconstructions may formally exclude a femoral head-neck junction curvature anomaly [58].

\section{Pincer-type FAI}

Found preferentially in middle-aged women [46, 48], this type of FAI is characterised by a morphological anomaly of the acetabulum causing excessive coverage of the femoral head: coxa profunda, acetabular protrusion or acetabular retroversion $[59,60]$. Acetabular anteversion, normally at an angle of $22.5 \pm 3$ degrees [61], is measured on an axial slice through the centre of the femoral head, however this angle may vary with pelvis flexion [62]. Rarely, it may be caused by trauma or labral ossification. Radiologically, retroversion manifests as the crossing sign and by the visibility of the ischial spine in the pelvis $[63,64]$. It causes direct contact between the anterior acetabular border and the femoral head-neck junction, thereby explaining preferential involvement of the labrum and a relatively preserved cartilage $[46,48,51]$. Later in the course, lesions to the postero-inferior cartilage by a contrecoup mechanism may appear [47]. Surgical management includes anterior acetabular osteoplasty for retroversion and circumferential osteoplasty in the case of a protrusion, in combination with labral lesion debridement [57]. Acetabular retroversion may be treated by periacetabular inverted osteotomy [60].

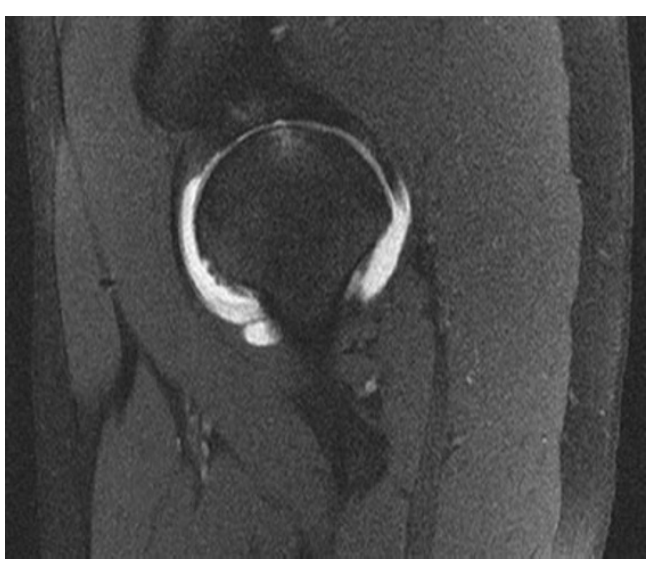

Fig. 13 Sagittal fat-saturated T1-weighted arthro-MR image shows a complete thinning of femoral and acetabular cartilage at the superior aspect of the joint associated with subchondral high-signal consistent with subchondral oedema 


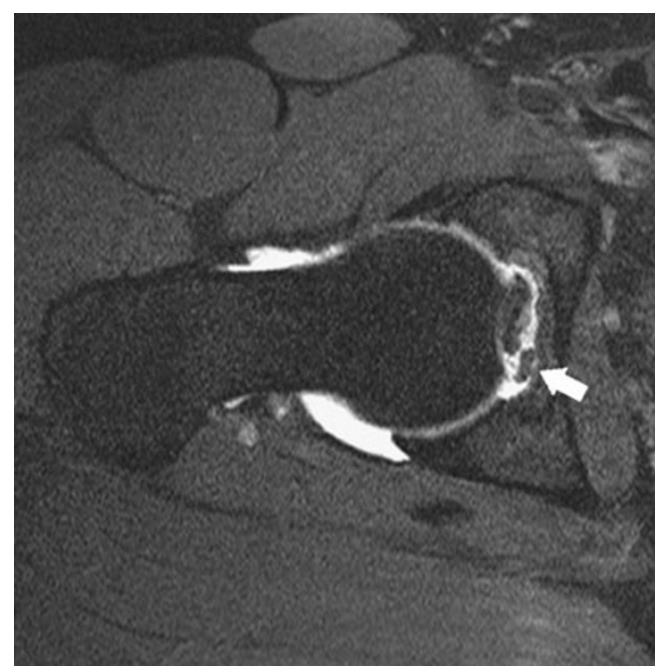

Fig. 14 Axial oblique fat-saturated T1-weighted arthro-MR image shows a hypointense structure wholly surrounded by contrast medium consistent with an intra-articular foreign body (white arrow

\section{Abnormality of the cartilage}

As the articular cartilage of the hip is thin, it is difficult to study with standard MR imaging. Sensitivity and specificity of MR arthrography in the detection of chondral lesions using T1-weighted spin-echo sequence are estimated to be about $62-79 \%$ and $77-94 \%[65,66]$ but multi-detector computed tomographic (MDCT) arthrography is superior to MR arthrography in terms of sensitivity, specificity and interobserver reproducibility [67]. Even if 3D double-echo steady-state sequence optimised for cartilage imaging improves lesion conspicuity, it doesn't seem to improve diagnostic performance [65]. As the articular cartilage is so thin and because of the direct apposition of the femur and acetabulum, it is sometimes difficult to differentiate femoral from acetabular cartilage. Inferior limb traction may improve articular cartilage analysis $[68,69]$, but it remains controversial [70]. Cartilage changes can be categorised into: normal, signal heterogeneity, fissuring, thinning to $<50 \%$ of the normal thickness, thinning to $>50 \%$ of the normal thickness and full thickness cartilage loss (Fig. 13) [71]. Early chondral anomalies are most often found in the antero-superior part of the joint, first on the acetabular side [66, 72]. Among 100 patients with mechanical symptoms, Neumann et al. [71] has estimated the prevalence of cartilage lesions to be $76 \%$, predominantly cartilage thinning to $<50 \%$. He also confirmed in vivo the association first reported in cadaveric studies [44] between labral tears and cartilage loss. Cartilage lesions are also correlated with bone marrow oedema [71]: analogous to the knee, bone marrow oedema may be indicative of more rapid progression [73] but has to be confirmed in longitudinal studies. Chondral lesions evolve to complete erosion of the articular cartilage, subchondral cyst formation and subchondral osteosclerosis. Before hip arthroscopy the aim of MR arthrogram is to assess the presence and severity of cartilage loss. It also could be useful in evaluating new chondroprotective or chondroregenerative drugs.

\section{Intra-articular foreign bodies}

The prevalence of intra-articular foreign bodies of the hip is low. They may be responsible for pain, locking, clicking and limited articular range of motion, and may or may not be associated with an osseocartilaginous defect. MR and MDCT arthrography allow for the differentiation of intraarticular foreign bodies from osteophytes, synovial folds or hypertrophic synovitis: classically, intra-articular foreign bodies are surrounded by contrast medium, but only partially in the case of osteophytes and synovial folds (Fig. 14) [74]. They are accessible to arthroscopic treatment.

\section{Abnormality of the ligamentum teres}

Abnormality of the ligamentum teres (ligament of the head of the femur) is rare. It may present as complete or partial
Fig. 15 a, b Complete tear of the ligamentum teres in an 29-year-old man. a Coronal reconstruction from a fatsaturated $3 \mathrm{D}$ gradient echo acquisition: the acetabular insertion of the ligamentum teres is interrupted. The ligamentum teres has an abnormal intermediate signal. b Sagittal fat-saturated T1-weighted arthroMR image through the acetabular bottom shows a laminated ligamentum teres completely vertically divided into two parts (white arrows)
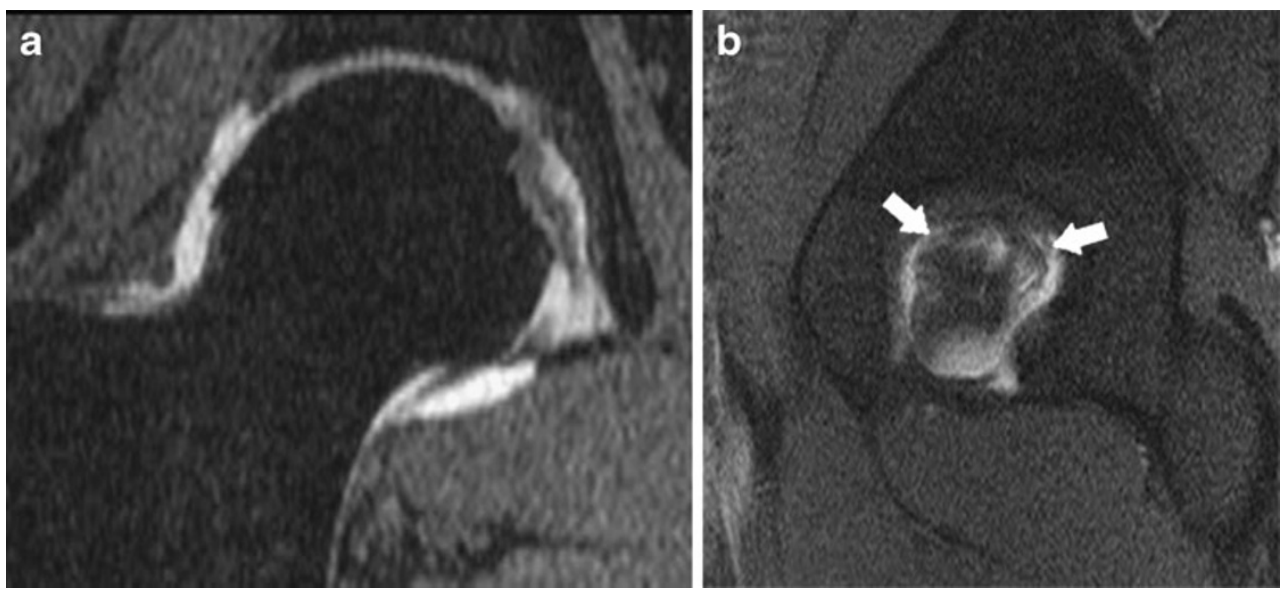
tear (Fig. 15). Degenerated ligamentum teres can be seen in young patients often with a history of avascular osteonecrosis or slipped capital femoral epiphysis in association with coxarthrosis [14, 75].

\section{Conclusion}

MR arthrography is a high performance imaging tool in the work-up of abnormalities of the acetabular labrum and articular cartilage, intra-articular foreign bodies and in femoroacetabular impingement. Although still an invasive procedure, because there is intra-articular injection of contrast medium, this imaging technique has shown excellent tolerance. Its indications have widened in the recent years since the development of conservative surgical interventions of the hip such as arthroscopy and surgery for femoroacetabular impingement.

\section{References}

1. Brown RR, Clarke DW, Daffner RH (2000) Is a mixture of gadolinium and iodinated contrast material safe during MR arthrography? AJR Am J Roentgenol 175(4):1087-1090

2. Duc SR, Hodler J, Schmid MR et al (2006) Prospective evaluation of two different injection techniques for MR arthrography of the hip. Eur Radiol 16(2):473-478

3. Andreisek G, Duc SR, Froehlich JM, Hodler J, Weishaupt D (2007) MR arthrography of the shoulder, hip, and wrist: evaluation of contrast dynamics and image quality with increasing injection-to-imaging time. AJR Am J Roentgenol 188(4):10811088

4. Wagner SC, Schweitzer ME, Weishaupt D (2001) Temporal behavior of intraarticular gadolinium. J Comput Assist Tomogr 25(5):661-670

5. Miller TT (2000) MR arthrography of the shoulder and hip after fluoroscopic landmarking. Skeletal Radiol 29(2):81-84

6. Graves MJ, Wakely S, Bearcroft PW et al (2008) MR-guided direct arthrography of the hip. J Magn Reson Imaging 28 (2):462-465

7. Guglielmi G, Biccari N, Mangano F, Toffanin R (2010) $3 \mathrm{~T}$ magnetic resonance imaging of the musculoskeletal system. Radiol Med. doi:10.1007/s11547-010-0521-4

8. Niitsu M, Nakai T, Ikeda K, Tang GY, Yoshioka H, Itai Y (2000) High-resolution MR imaging of the knee at 3 T. Acta Radiol 41 (1):84-88

9. Ramnath RR (2006) 3T MR imaging of the musculoskeletal system (Part II): clinical applications. Magn Reson Imaging Clin N Am 14(1):41-62

10. Peterson DM, Carruthers CE, Wolverton BL et al (1999) Application of a birdcage coil at 3 Tesla to imaging of the human knee using MRI. Magn Reson Med 42(2):215-221

11. Ryan M, Cunningham P, Cantwell C, Brennan D, Eustace S (2005) A comparison of fast MRI of hips with and without parallel imaging using SENSE. Br J Radiol 78(928):299-302

12. Plotz GM, Brossmann J, von Knoch M, Muhle C, Heller M, Hassenpflug J (2001) Magnetic resonance arthrography of the acetabular labrum: value of radial reconstructions. Arch Orthop Trauma Surg 121(8):450-457
13. Kubo T, Horii M, Harada Y et al (1999) Radial-sequence magnetic resonance imaging in evaluation of acetabular labrum. J Orthop Sci 4(5):328-332

14. Hong RJ, Hughes TH, Gentili A, Chung CB (2008) Magnetic resonance imaging of the hip. J Magn Reson Imaging 27(3):435-445

15. Saupe N, Zanetti M, Pfirmann CW, Wels T, Schwenke C, Hodler J (2009) Pain and other side effects after MR arthrography: prospective evaluation in 1085 patients. Radiology 250(3):830-838

16. Hugo PC 3rd, Newberg AH, Newman JS, Wetzner SM (1998) Complications of arthrography. Semin Musculoskelet Radiol 2 (4):345-348

17. Watanabe W, Itoi E, Yamada S (2002) Early MRI findings of rapidly destructive coxarthrosis. Skeletal Radiol 31(1):35-38

18. Wyler A, Bousson V, Bergot C et al (2007) Hyaline cartilage thickness in radiographically normal cadaveric hips: comparison of spiral CT arthrographic and macroscopic measurements. Radiology 242(2):441-449

19. Engel A, Hajek PC, Kramer J (1990) Magnetic resonance knee arthrography. Acta Orthop Scand 61(Suppl 240):1-47

20. Keene GS, Villar RN (1994) Arthroscopic anatomy of the hip: an in vivo study. Arthroscopy 10(4):392-399

21. Tschauner C, Hofmann S, Czerny C (1997) Hip dysplasia. Morphology, biomechanics and therapeutic principles with reference to the acetabular labrum. Orthopade 26(1):89-108

22. Hodler J, Yu JS, Goodwin D, Haghighi P, Trudell D, Resnick D (1995) MR arthrography of the hip: improved imaging of the acetabular labrum with histologic correlation in cadavers. AJR Am J Roentgenol 165(4):887-891

23. Edwards DJ, Lomas D, Villar RN (1995) Diagnosis of the painful hip by magnetic resonance imaging and arthroscopy. J Bone Joint Surg Br 77(3):374-376

24. Petersilge CA, Haque MA, Petersilge WJ, Lewin JS, Lieberman JM, Buly R (1996) Acetabular labral tears: evaluation with MR arthrography. Radiology 200(1):231-235

25. Williams PL, Warwick R (1980) Arthrology: the joints of the lower limb - the hip (coaxial) joint. Saunders, Philadelphia

26. Czerny C, Hofmann S, Urban M et al (1999) MR arthrography of the adult acetabular capsular-labral complex: correlation with surgery and anatomy. AJR Am J Roentgenol 173(2):345-349

27. Aydingoz U, Ozturk MH (2001) MR imaging of the acetabular labrum: a comparative study of both hips in 180 asymptomatic volunteers. Eur Radiol 11(4):567-574

28. Cotten A, Boutry N, Demondion X et al (1998) Acetabular labrum: MRI in asymptomatic volunteers. J Comput Assist Tomogr 22(1):1-7

29. Abe I, Harada Y, Oinuma K et al (2000) Acetabular labrum: abnormal findings at MR imaging in asymptomatic hips. Radiology 216(2):576-581

30. Lecouvet FE, Vande Berg BC, Malghem J et al (1996) MR imaging of the acetabular labrum: variations in 200 asymptomatic hips. AJR Am J Roentgenol 167(4):1025-1028

31. Studler U, Kalberer F, Leunig M et al (2008) MR arthrography of the hip: differentiation between an anterior sublabral recess as a normal variant and a labral tear. Radiology 249(3):947-954

32. Dinauer PA, Murphy KP, Carroll JF (2004) Sublabral sulcus at the posteroinferior acetabulum: a potential pitfall in MR arthrography diagnosis of acetabular labral tears. AJR Am J Roentgenol 183 (6): 1745-1753

33. Czerny C, Hofmann S, Neuhold A et al (1996) Lesions of the acetabular labrum: accuracy of MR imaging and MR arthrography in detection and staging. Radiology 200(1):225-230

34. Dameron TB (959) Bucket-handle tear of acetabular labrum accompanying posterior dislocation of the hip. 141:131-134

35. Dorrell JH, Catterall A (1986) The torn acetabular labrum. J Bone Joint Surg Br 68(3):400-403

36. Fitzgerald RH Jr (1995) Acetabular labrum tears. Diagnosis and treatment. Clin Orthop Relat Res 311:60-68 
37. Mintz DN, Hooper T, Connell D, Buly R, Padgett DE, Potter HG (2005) Magnetic resonance imaging of the hip: detection of labral and chondral abnormalities using noncontrast imaging. Arthroscopy 21(4):385-393

38. Ghebontni L, Roger B, El-khoury J, Brasseur JL, Grenier PA (2000) MR arthrography of the hip: normal intra-articular structures and common disorders. Eur Radiol 10(1):83-88

39. Leunig M, Werlen S, Ungersbock A, Ito K, Ganz R (1997) Evaluation of the acetabular labrum by MR arthrography. J Bone Joint Surg Br 79(2):230-234

40. Blankenbaker DG, De Smet AA, Keene JS, Fine JP (2007) Classification and localization of acetabular labral tears. Skeletal Radiol 36(5):391-397

41. Lage LA, Patel JV, Villar RN (1996) The acetabular labral tear: an arthroscopic classification. Arthroscopy 12(3):269-272

42. Magee T, Hinson G (2000) Association of paralabral cysts with acetabular disorders. AJR Am J Roentgenol 174(5):1381-1384

43. Kramer J, Recht MP (2002) MR arthrography of the lower extremity. Radiol Clin North Am 40(5):1121-1132

44. McCarthy JC, Busconi B (1995) The role of hip arthroscopy in the diagnosis and treatment of hip disease. Orthopedics 18(8):753-756

45. Beaule PE, Zaragoza E, Copelan N (2004) Magnetic resonance imaging with gadolinium arthrography to assess acetabular cartilage delamination. A report of four cases. J Bone Joint Surg Am 86-A(10):2294-2298

46. Beck M, Leunig M, Parvizi J, Boutier V, Wyss D, Ganz R (2004) Anterior femoroacetabular impingement: part II. Midterm results of surgical treatment. Clin Orthop Relat Res 418:67-73

47. Bredella MA, Stoller DW (2005) MR imaging of femoroacetabular impingement. Magn Reson Imaging Clin N Am 13(4):653-664

48. Ganz R, Parvizi J, Beck M, Leunig M, Notzli H, Siebenrock KA (2003) Femoroacetabular impingement: a cause for osteoarthritis of the hip. Clin Orthop Relat Res(417):112-120

49. Tanzer M, Noiseux N (2004) Osseous abnormalities and early osteoarthritis: the role of hip impingement. Clin Orthop Relat Res 429:170-177

50. Tannast M, Siebenrock KA, Anderson SE (2007) Femoroacetabular impingement: radiographic diagnosis-what the radiologist should know. AJR Am J Roentgenol 188(6):1540-1552

51. Beck M, Kalhor M, Leunig M, Ganz R (2005) Hip morphology influences the pattern of damage to the acetabular cartilage: femoroacetabular impingement as a cause of early osteoarthritis of the hip. J Bone Joint Surg Br 87(7):1012-1018

52. Notzli HP, Wyss TF, Stoecklin CH, Schmid MR, Treiber K, Hodler J (2002) The contour of the femoral head-neck junction as a predictor for the risk of anterior impingement. J Bone Joint Surg Br 84(4):556-560

53. Rakhra KS, Sheikh AM, Allen D, Beaule PE (2009) Comparison of MRI alpha angle measurement planes in femoroacetabular impingement. Clin Orthop Relat Res 467(3):660-665

54. Kassarjian A, Yoon LS, Belzile E, Connolly SA, Millis MB, Palmer WE (2005) Triad of MR arthrographic findings in patients with camtype femoroacetabular impingement. Radiology 236(2):588-592

55. Leunig M, Beck M, Kalhor M, Kim YJ, Werlen S, Ganz R (2005) Fibrocystic changes at anterosuperior femoral neck: prevalence in hips with femoroacetabular impingement. Radiology 236(1):237-246

56. Ganz R, Leunig M, Leunig-Ganz K, Harris WH (2008) The etiology of osteoarthritis of the hip: an integrated mechanical concept. Clin Orthop Relat Res 466(2):264-272

57. Lavigne M, Parvizi J, Beck M, Siebenrock KA, Ganz R, Leunig M (2004) Anterior femoroacetabular impingement: part I. Techniques of joint preserving surgery. Clin Orthop Relat Res 418:61-66
58. Neumann M, Cui Q, Siebenrock KA, Beck M (2009) Impingement-free hip motion: the 'normal' angle alpha after osteochondroplasty. Clin Orthop Relat Res 467(3):699-703

59. Ruelle M, Dubois JL (1962) The protrusive malformation and its arthrosic complication. I. Radiological and clinical symptoms. Etiopathogenesis. Rev Rhum Mal Osteoartic 29:476-489

60. Siebenrock KA, Schoeniger R, Ganz R (2003) Anterior femoroacetabular impingement due to acetabular retroversion. Treatment with periacetabular osteotomy. J Bone Joint Surg Am 85-A (2): $278-286$

61. Frot B, Duparc J (1973) Radiologic measurement of anteversion, inclination and depth of the acetabulum. J Radiol Electrol Med Nucl 54(3):213-222

62. Aubry S, Marinescu A, Forterre O, Runge M, Garbuio P (2005) Definition of a reproducible method for acetabular anteversion measurement at CT. J Radiol 86(4):399-404

63. Jamali AA, Mladenov K, Meyer DC et al (2007) Anteroposterior pelvic radiographs to assess acetabular retroversion: high validity of the "cross-over-sign". J Orthop Res 25(6):758-765

64. Kalberer F, Sierra RJ, Madan SS, Ganz R, Leunig M (2008) Ischial spine projection into the pelvis: a new sign for acetabular retroversion. Clin Orthop Relat Res 466(3):677-683

65. Knuesel PR, Pfirrmann CW, Noetzli HP et al (2004) MR arthrography of the hip: diagnostic performance of a dedicated water-excitation 3D double-echo steady-state sequence to detect cartilage lesions. AJR Am J Roentgenol 183(6):1729-1735

66. Schmid MR, Notzli HP, Zanetti M, Wyss TF, Hodler J (2003) Cartilage lesions in the hip: diagnostic effectiveness of MR arthrography. Radiology 226(2):382-386

67. Nishii T, Tanaka H, Nakanishi K, Sugano N, Miki H, Yoshikawa $\mathrm{H}$ (2005) Fat-suppressed 3D spoiled gradient-echo MRI and MDCT arthrography of articular cartilage in patients with hip dysplasia. AJR Am J Roentgenol 185(2):379-385

68. Llopis E, Cerezal L, Kassarjian A, Higueras V, Fernandez E (2008) Direct MR arthrography of the hip with leg traction: feasibility for assessing articular cartilage. AJR Am J Roentgenol 190(4):1124-1128

69. Nakanishi K, Tanaka H, Nishii T, Masuhara K, Narumi Y, Nakamura H (1999) MR evaluation of the articular cartilage of the femoral head during traction. Correlation with resected femoral head Acta Radiol 40(1):60-63

70. Wettstein M, Guntern D, Theumann N (2008) Direct MR arthrography of the hip with leg traction: feasibility for assessing articular cartilage. AJR Am J Roentgenol, 191(5):W206; author reply W207

71. Neumann G, Mendicuti AD, Zou KH et al (2007) Prevalence of labral tears and cartilage loss in patients with mechanical symptoms of the hip: evaluation using MR arthrography. Osteoarthritis Cartilage 15(8):909-917

72. Noguchi Y, Miura H, Takasugi S, Iwamoto Y (1999) Cartilage and labrum degeneration in the dysplastic hip generally originates in the anterosuperior weight-bearing area: an arthroscopic observation. Arthroscopy 15(5):496-506

73. Felson DT, Chaisson CE, Hill CL et al (2001) The association of bone marrow lesions with pain in knee osteoarthritis. Ann Intern Med 134(7):541-549

74. Kramer J, Laub G, Czerny C, Recht M (2006) MR and MR arthrography in imaging of the hip and bony pelvis. Springer, Berlin Heidelberg

75. Gray AJ, Villar RN (1997) The ligamentum teres of the hip: an arthroscopic classification of its pathology. Arthroscopy 13 (5):575-578 